\title{
Neurovascular Centers-A Need for a Change
}

In the last two decades two developments have been celebrated as major improvements in the treatment of acute ischemic stroke: the nationwide implementation of stroke units and the development of iv thrombolysis.

This universal concept has been strongly supported by the stroke community and health systems resulting in a significant increase in stroke units with a respectable reimbursement for treated stroke patients. However, the benefit of iv thrombolysis in severe ischemic stroke patients is only minor and specially in smaller stroke units the rate of iv thrombolysis is lower than expected. In contrast the rate of patients with severe stroke transferred to larger hospitals with neurointerventional units has decreased due to various reasons. This has led to the opinion that with this concept of widespread distribution of stroke units, patients with severe stroke become increasingly less optimally treated and get lost in the peripheral.

An answer to this problem is thought to be the foundation of neurovascular centers which can support smaller stroke units in the selection of patients for more aggressive stroke treatment. The significant improvements in endova- scular stroke treatment with high recanalization rates up to $90 \%$ and good clinical outcome even in patients with severe stroke are the major motor for this idea. However, this kind of stroke treatment needs a centralization of treatment to offer high quality in experienced hands on a $24 \mathrm{~h} / 7$ day basis. Widespread use of endovascular stroke treatment in inexperienced hands has to be avoided but the creation of certified neurovascular centers which have close connections to peripheral stroke units offers the chance that this useful treatment can be supplied within acceptable time windows to all stroke patients who are suitable for the endovascular approach.

Recent discussion has shown that there will be some resistance to this new concept but in the respect of our patients it is worth going this way and to demand this new approach with the building of neurovascular centers in our health systems.

Prof. Dr. Olav Jansen

President of the German Society of Neuroradiology 